\title{
Editorial
}

\section{To build a brand, use something old as a link to something new}

Brand positioning seems to work best when we are very single minded and very consistent - even at the expense of being very repetitive with brand communications.

A problem with giving people new information about a brand is that the new information tends to interfere with the old - or interfere with the mental availability of the old. $^{1,2}$

Too often brands try to be everything to everybody. There is a great temptation to claim excellence not just on one key attribute but to claim it on that one and then another and then another. This may be in the same commercial or in several executions but the result is usually the same. Experience from tracking hundreds of campaigns over the past decade indicates that this message proliferation rarely meets with success. ${ }^{2}$

This is not just the case with low involvement products. The same can be observed with high involvement products as well. The triggering of a brand in peoples' minds should automatically bring with it strong associations of the brand's key positioning attribute(s).

In order to maximise mental availability we need to minimise message proliferation. It is when a brand is much more single minded about the image attribute(s) it wishes to 'own' that brand positioning seems to work best.

Things and times change, however, and brand managers do eventually find themselves in the position where they feel they have to provide new information or focus on new attributes or they find they have to introduce new variants of the brand.

The question is how do you move on and say something new about your brand while minimising this interference effect? How do you keep the benefits of what you have already communicated, but still get the benefits of communicating the new information.

There are two main ways by which new information can be presented while minimising or offsetting the interference effect.

One way is to choose an appropriate name for the brand in the first place that locks in the key image attribute such as:

- Lean Cuisine - weight control;

- Diet Coke — weight control;

- Healthy Choice — healthy.

Brands like these lock the key attribute into the name itself. So the mental connection with the brand is not as subject to interference from newer information that comes along. The association gets repetitively reinforced by the mere mention of the name.

When 'Diet Coke' was launched, its claim to health and weight control was staked out implicitly in the name. At the same time its slogan was able to make a grab for the 'taste' position, ie 'Diet Coke - Just for the taste of it!'.

The 'weight control' association was linked more inherently and more linguistically to the brand by the name itself much more than was its predecessor, 'TAB'.

So this is one strategy - to provide a brand with a bit more scope to move by virtue of its name.

\section{INTEGRATIVE LINKS}

Another way is to establish inter-connecting mental links.

Let me take as an illustrative example 

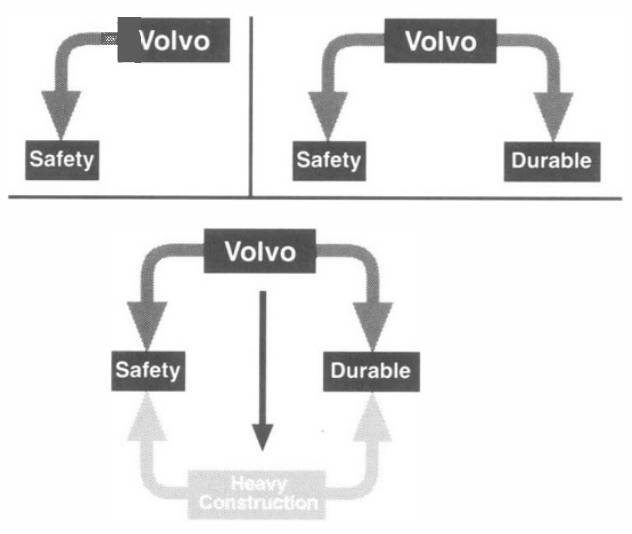

Volvo. This is a brand of car that has firmly established a safety image over many years in various parts of the world. Let us imagine you as brand manager for Volvo want to broaden its positioning and now move on to emphasise 'durability'. What should you do? You would be ill-advised just simply to switch to a durability platform.

Rather than simply asserting that 'Volvo lasts longer than other cars', the trick is to look for some potential interconnecting mental link between the new and the old a link between the attribute that you already own, 'safety', and the new attribute, 'durability'.

Try to link the new to the old by using something like:

'You know Volvo for its safety. One reason Volvo is safer is its heavier construction. But because of this heavier construction it is also more durable. Volvo - the car that outlasts other cars.'

This has the advantage of reinforcing the old position, safety, while communicating the new.

The interconnecting link means that the two positions are mutually self-supporting in buyers' minds. (Thinking of one attribute tends to trigger the other.)
By forging additional mental connections that are not just connections to the brand name, the resultant mental structure becomes a more integrated and more mutually reinforcing whole.

\section{SEARCHING FOR CONNECTORS}

Additional connections can be between the image attributes themselves or between the brand and an attribute and its rationale (the reason why). In the mind there are all sorts of potential interconnections between product categories (eg toothbrushes and toothpaste), between brands (eg Coca-Cola and Diet Coke) and between attributes (eg high price - high quality).

Less effective - but better than none is to use some common element like a high profile presenter to link them, or to link them via a unique executional style that your brand can 'own'.

The point is that integration is a key to facilitating memory and successfully communicating new information about a brand. Build as many links or connections as possible between the pieces of information themselves so then each one can act as a retrieval cue for the other.

\section{MENTAL PAPER CLIPS}

It is like filing something in a file. The more things in the file the longer it will take us to retrieve any particular thing we are looking for - simply because of the clutter. But if the file is organised so that the new is paper clipped to the old, it makes the retrieval task that much easier - and quicker. Associative links are like mental paper clips.

Managers of brands should not have to be content with unintegrated message strategies. It is much better to look for ways to build-on existing connections and forge associative links between these messages themselves. Whether it be two brand vari- 
ants, or two image attributes, just relying the common label, the brand, to 'paper clip' them together in memory is not a good strategy.

\section{References:}

(1) Anderson John, R. (1990) 'Cognitive Psychology and its Implications', W. H. Freeman and Co, NY, pp. 164-170.

(2) Sutherland, Max (1993) 'Advertising and the Mind of the Consumer: What Works, What Doesn't and Why', Allen \& Unwin, Australia, pp. 126-131.
Max Sutherland Editorial Board 